\title{
Artemisinin Derivatives Based Therapeutic Combinations for the Treatment of Uncomplicated Plasmodium Falciparum Malaria: Find-Ings of a Meta-Analysis of Surveys Carried Out in Senegal
}

\author{
Papa Ndiaye*1, Annie Wassiatou Abiola ${ }^{2}$, Roger Clement Tine ${ }^{2}$, Kouvidjin Ekoue ${ }^{2}$, Coumba Cisse ${ }^{2}$, Khadime Sylla $^{2}$ \\ , Doudou Sow ${ }^{2}$, Ibrahima Diallo ${ }^{3}$, Thwing J ${ }^{4}$, Jean Louis Ndiaye ${ }^{2}$, Magatte Ndiaye ${ }^{2}$, Aminata Collé Lo $^{2}$, Oumar Gaye $^{2}$ \\ and Babacar Faye ${ }^{2}$ \\ ${ }^{1}$ Section Santé Publique, Unité de Formation et de Recherche des sciences de la Santé (UFR2S), Université Gaston Berger (UGB) Saint-Louis, Sénégal \\ ${ }^{2}$ Service de Parasitologie - Mycologie, Faculté de Médecine, Pharmacie et Odontologie, UCAD, Sénégal \\ ${ }^{3}$ Programme National de Lutte contre le Paludisme, Ministère de la Santé et de l'Action Sociale, Sénégal \\ ${ }^{4}$ USAID PMI, Sénégal
}

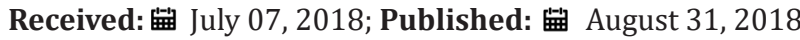

*Corresponding author: Papa Ndiaye, Section Santé Publique, Unité de Formation et de Recherche des sciences de la Santé (UFR2S), Université Gaston Berger (UGB) Saint-Louis, Senegal

\begin{abstract}
Objectives: The objective of this study was to analyze studies comparing efficacy of various Artemisinin-based combination therapies (ACTs).

Study Design: Meta-analysis of 11 selected studies: 10 were comparative with AL and 1 without AL.

Method: The patient groups were comparable at the inclusion. Patients were followed for 28 days (D) according to the WHO protocol with regular clinical and laboratory evaluations.

Results: Very good efficacy ( $>95 \%$ ) were obtained at D28, for all the combinations tested, with no statistically significant difference. The total clearance of fever was obtained at D3 for all patients. The fever was more persistent at D2 in children with ASAQ and ASMQ than in those with AL. Parasitic clearance was better for all ACTs than for AL, except for DHPQTMP. At D2, gametocyte carriage was similar for the treatment groups analyzed. Side effects were more marked in patients treated with ASMQ than in those on AL.
\end{abstract}

Conclusion: ACTs are of great benefit in the management of uncomplicated P. falciparum malaria. The ASAQ, ASMQ, DHAPQ and DHPQTMP associations are as effective and well tolerated as the AL association.

\section{Introduction}

As the largest human parasitic disease globally, malaria is a significant public health problem [1-3]. In the world, $40 \%$ of the population are at risk of malaria infection. Programs have reduce the incidence of the disease and the associated mortality by $30 \%$ and $47 \%$ respectively between 2000 and 20134 . However, although progress has been good, a lot remains to be done; 198 million cases of malaria and 584000 associated deaths still occur every year [4]. Early and proper treatment of cases is one of the pillars of malaria control. It has unfortunately come up against the emergence of resistance to the anti-malaria molecules used in mono-therapies so far. The WHO had therefore recommended bi-therapies with artimisinin combination therapies (ACTs) $[5,6]$ and asked countries to ensure a permanent monitoring of their effectiveness [7].
In Africa, most cases and fatalities occur in non-immunized persons, pregnant women and children. Every minute, a child dies of malaria. That represents $22 \%$ of all child deaths. But, with an increase in funds for malaria control, RDTs and ACTs has grown from $68 \%$ in 2005 to $75 \%$ in 2011, especially in sub-Saharan Africa. In Senegal, malaria related morbidity has dropped from $33.57 \%$ in 2006 to $3.07 \%$ in 2009. The National Malaria Control Program (PNLP) adopted ACTs in 2006 as the recommended treatment for uncomplicated malaria with Plasmodium falciparum [8]. Back then, Artemether-Lumefantrin and Artesunate-Amodiaquin were used as first line therapy. In 2010, the combination Dihydro-ArtemisininPiperaquin was introduced to respond to the ACT stock-outs which were observed countrywide. That scale-up included also RDTs 
and it was accompanied by the setting up of a pharmacovigilance system [9] and an annual plan for the surveillance of the sensitivity of P. falciparum to ACTs under which several clinical trials were performed. The objective of this meta-analysis was to compare dthe clinical and parasitological effectiveness, the thermal and parasitic clearance, lethe gametocyte carriage and the tolerance level of various ACTs used to treat uncomplicated P. falciparum malaria in Senegal.

\section{Method}

\section{Type of Study}

This retrospective meta-analysis study covered a number of comparative studies aimed at assessing the effectiveness and safety of artimisinin-based combination therapies (ACTs). A review of the literature available on PubMed, Embase, Google Scholar and ClinicalTrials.gov was performed in july 2015 using four key words (malaria, ACTs, clinical trials, Senegal). It allowed for the identification of all clinical trial reports published since 2000 in relation to ACTs.

\section{Criteria for the Selection of Studies Covered}

There were six selection criteria: Assessment of the effectiveness of one or several ACTs; sampling for parasitemia at least once a day on days 0, 1, 2 and 3 after beginning of treatment; carried out in Senegal; favourable opinion of the National Ethics Committee Health Research (CNERS); implementation of the WHO protocole (2001, 2003 or 2004); subject of the publication.

\section{Sites}

All the studies analyzed were carried out in four health districts (Guédiawaye, Kaolack, Ndoffane, Pikine, Touba), which is representative of the various forms of malaria in Senegal.

\section{Common Protocole Followed}

The studies involved persons diagnosed with uncomplicated malaria based on clinical signs (fever $\otimes 37^{\circ} 5 \mathrm{C}$ with or without chills, headaches, muscle soreness, diffuse pains algies diffuses) and confirmed by isolation of P. falciparum in the blood. There were five criteria for the inclusion of patients: $\nabla(1)$ no pregnancy or breastfeeding ; $\nabla(2)$ ability to take medication per os ; $\otimes(3)$ initial parasite density between 1000 and 100000 asexual forms $/ \mu \mathrm{l}$ in the blood; $\nabla(4)$ no record of hypersensitivity to the drugs to be assessed; $\otimes(5)$ informed parental consent. There were four non- inclusion criteria: $\nabla(1)$ mixed infestation or mono specific infestation by another type of Plasmodium; $\otimes(2)$ severe malnutrition (child below $-3 \mathrm{z}$ score with a symmetrical edema affecting at least both legs and mid-upper arm circumference $<110 \mathrm{~mm}$ ); $\nabla(3)$ intake of medication likely to affect antimalarial pharmacokinetics; $\bigotimes(4) \bigotimes$ malaria intake of antimalarial medication within the 15 days preceding the study, hypersensitivity to or contraindication of any of the drugs involved.

There were four criteria for discontinuation of monitoring: $\nabla(1)$ withdrawal of patient consent; $\nabla(2)$ serious adverse events; (3) unsatisfactory response to therapy; $\nabla(4)$ violation of the protocole (because of an event which prevents a continuous and specific interpretation of a patient's response to treatment, mixed infestation in the course of the study, selfmedication, lost of follow up, death.

\section{Data Analysis for the Meta- Analysis}

Sociodemographic (age, gender and geographical area), clinical (clinical signs and temperature) and biological (parasitemia) data were extracted from various clinical trial reports for analysis and comparison.

\section{Statistical Analysis}

Based on PDF documents, the basic details of the methodology used, the assessed treatment arms and the studied areas locations were documented. The year considered was the year during which the document was published. The start and end dates for the recruitment of the patients were also recorded when available. For the purposes of statistical analysis, the main criterion used was positive microscopy on days 1,2 , and 3 and on the other days of the monitoring period. The proportions of patients who remained parasitemic, expressed in terms of failure rate, were defined separately for each study based on individual patient data. Statistical heterogeneity was quantified as the variance of random effects using the maximum-likelyhood method. The initial variables taken into consideration included age, gender, fever (temperature > $37.5^{\circ} \mathrm{C}$ ), parasitemia, gametocyte carriage, treatment method and year of study. The continuous variables of the different groups were compared using a linear regression model. The data which are not usually issued were compared using the Mann-Whitney test or the Kruskal-Wallis test. All this statistical analysis was done with the R software (version 3.1.2, R Foundation for Statistical Computing, Vienna, Austria).

\section{Results}

\section{General Layout of the Survey}

Out of 12 studies listed, one was ruled out and 11 were kept (4005 patients). The 10 (3691 patients) were comparisons between Arthemeter-lumefantrine (1340 patients) and one or two other ACTs (2351 patients): ॠArtesunate-Mefloquine (ASMQ) for adults ; \Artesunate-Mefloquine (ASMQ) for children; $\triangle$ ArtesunateAmodiaquine (ASAQ) for adults; $\triangle$ Artesunate-Amodiaquine (ASAQ) for children; $\bowtie$ Dihydro-artemisinine-Piperaquine (DHAPQ); $\bigotimes$ Dyhydro-artemisine-piperaquinetrimethoprine (DHAPQ-TMP), $\triangle$ ASAQ repeated ; $\triangle$ ASAQ and ASMQ; $\triangle A S A Q$ and DHAPQ ; $\triangle \mathrm{ASAQ}$ fixed dose and two doses. The last study assessed ASAQ in a single daily dose versus a divided dose twice a day. It involved 314 patients (Tables 1 \& 2).

\section{Sociodemographic Features}

Patients age varied between 5 and 13 years for children and between 16 and 25 years for adults under AL. It varied between 4 and 15 years for children and between 16 and 25 years among adults treated with the other ACTs. The weights were between 16 and $58 \mathrm{~kg}$ for the patients treated with AL against 15 to $62 \mathrm{~kg}$ for those treated with the other ACTs. The gender ration (male/female) was 1.3 . 
Table 1: List of Studies and Codes.

\section{Excluded study}

Efficacy and safety of a fixed-dose oral combination of pyronaridine-artesunate compared with artemether lumefantrine in children and adults with Uncomplicated Plasmodium falciparum malaria: a randomized non-inferiority trial. Lancet 2010;375: 1457-67.

\section{Included studies}

1) Faye B, Ndiaye JL, Ndiaye D, Dieng Y, Faye O, Gaye O. Efficacy and tolerability of four antimalarial-combinations in the treatment of uncomplicated Plasmodium falciparum malaria in Senegal. Malaria Journal 2007, 6: 80 doi:10.1186/1475-2875-6-80. (FAYE_ASAQ_AL_2007)

2) Ndiaye JLA, Faye B, Diouf AM, Kuété T, Cisse M, et al. Randomized, comparative study of the efficacy and safety of artesunate plus amodiaquine, administered as a single daily intake versus twodailyintakes in the treatment of uncomplicated falciparum malaria. Malaria Journal 2008, 7:16 doi:10.1186/1475-2875-7-16. (NDIAYE_2008)

3) Ndiaye JL, Randrianarivelojosia M, Sagara I, Brasseur P, Ndiaye I, Faye B, et al. Randomized, multicen-ter assessment of the efficacy and safety of ASAQ - a fixed-dose artesunate-amodiaquinecombinationtherapyin the treatment of uncomplicated Plasmodium falciparum malaria.

4) Efficacy and tolerability of artesunate-amodiaquine (Camoquinplus) versus artemether-lumefantrine (Coartem) against uncomplicated Plasmodium falciparum malaria: multisite trial in Senegal and Ivo-ryCoast. Malaria Journal 2010. (FAYE_ASAQ_AL_2010)

5) Faye B, Ndiaye JL, Tine R, Sylla K, Gueye A, Lô AC, Gaye O. A Randomized Trial of Artesunate Meflo-quine versus Artemether Lumefantrine for the Treatment of Uncomplicated Plasmodium falciparum-Malaria in Senegalese Children. Malaria Journal 2010. (FAYE_ASMQ_AL_2010)

6) Ndiaye JLA, Faye B, Gueye A, Tine R, Ndiaye D, Tchania C, et al. Repeated treatment of recurren-tuncomplicated Plasmodium falciparum malaria in Senegal with fixed-dose artesunate plus amodia-quine versus fixed-dose artemether plus lumefantrine: a randomized, open-label trial. Malaria Journal 2011. (NDIAYE_ASAQ_AL_2011)

7) Yavo W, Faye B, Kuete T, Djohan V, Oga SA, Kassi RR, et al. Multicentric assessment of the efficacy and tolerability of dihydroartemisininpiperaquinecompared to artemether-lumefantrine in the treat-ment of uncomplicated Plasmodium falciparum malaria in sub-SaharanAfrica. Malaria Journal 2011. (YAVO_DHAPQ_AL_2011)

8) Menan H, Faye O, Same-Ekobo A, Oga ASS, Faye B, Kiki-Barro CP, et al. Comparative study of the effi-cacy and tolerability of dihydroartemisinin piperaquine - trimethoprim versus artemether - lu-mefantrine in the treatment of uncomplicated Plasmodium falciparum malaria in Cameroon, IvoryCoast and Senegal. Malaria Journal 2011. (MENAN_DHAPQ_AL_2011)

9) Faye B, Kuété T, Kiki-Barro CP, Tine RC, Nkoa T, Ndiaye JLA, et al. Multicentre study evaluating the non-inferiority of the new pediatric formulation of artesunate/amodiaquine versus artemether / lu-mefantrine for the management of uncomplicated Plasmodium falciparum malaria in children in Cameroon, Ivory Coast and Senegal. Malaria Journal 2012. (FAYE_ASAQ_AL_2012)

10) Tine RCK, Faye B, Sylla K, Ndiaye JL, Ndiaye M, Sow D, et al. Efficacy and tolerability of a new formu-lation of artesunate-mefloquine for the treatment of uncomplicated malaria in adult in Senegal: open randomized trial. Malaria Journal 2012, 11: 416. (TINE_ASMQ_AL_2012)

11) Sylla K, Abiola A, Tine RKC, Faye B, Sow D, Ndiaye JL, et al. Monitoring the efficacy and safety of three artemisinin based-combinations therapies in Senegal: results from two years surveillance. Malaria journal 2013. (SYLLA_ASAQ_AL_DHAPQ_2013)

Table 2: Sample Size by Type of Analysis Performed in Each Study.

\begin{tabular}{|c|c|c|c|}
\hline \multicolumn{2}{|c|}{ Comparative studies } & Intent To Treat & Per Protocole \\
\hline & AL & 1340 & 1036 \\
\cline { 2 - 4 } & ASAQ & 1548 & 305 \\
\cline { 2 - 4 } & ASMQ & 462 & 238 \\
\cline { 2 - 4 } & DHAPQ & 256 & 83 \\
\cline { 2 - 4 } & DHAPQT & 85 & 2553 \\
\cline { 2 - 4 } & Subtotal & 3691 & 148 \\
\hline \multirow{3}{*}{ Without AL } & ASAQ 1 dose & 159 & 150 \\
\cline { 2 - 4 } & ASAQ 2 doses & 155 & 298 \\
\hline & Sub-total & 314 & 2851 \\
\hline
\end{tabular}

\section{Initial Clinical and Biological Signs}

Initially, all patients involved (adults and children) had hyperthermia and a parasite density between 1000 and 100.000 . The average recorded temperature for the studies involving $\mathrm{AL}$ varied between $37.7^{\circ} \mathrm{C}$ and $38.5^{\circ} \mathrm{C}$ for the $\mathrm{AL}$ group and $37.9^{\circ} \mathrm{C}$ àto $38.8^{\circ} \mathrm{C}$ for the others; it was $38.3^{\circ} \mathrm{C}$ for the comparative study without AL. For studies involving AL, parasitemia (parasites/ $\mu \mathrm{l})$ varied between 13212 à 37370 for AL-treated patients and between 10840 and 41863 for those treated with other ACTs. It ranged between 13750 and 16421 for the patients involved in the comparative study without AL.

\section{Therapeutic Efficacy}

Adequate clinical and parasitological response (ACPR) on day28: the data was collected from six studies. In the intent-totreat analysis, APCR was higher for DHPQ, ASMQ for adults, ASAQ for children and ASAQ for adults than for $\mathrm{AL}$; but the differences 
were not statistically significant (Figure 1 ). On a per protocole basis (PP), the ACPR was higher with ASAQ for children and ASAQ for adults than with routine ASAQ and it was identical between AL and DHAPQ and ASMQ; the difference was not significant (Figure 2). Therapeutic failures, which were more frequent in ASAQ-treated patients showed almost identical proportions as ACTs et AL.

\begin{tabular}{|c|c|c|c|}
\hline Study ID & & ES $(95 \% \mathrm{CI})$ & $\begin{array}{c}\% \\
\text { Weight }\end{array}$ \\
\hline FAYE_ASAQ_AL_2010 & - & $1.00(-0.92 ; 2.92)$ & 14.45 \\
\hline FAYE_ASAQ_AL_2012 & + & \multicolumn{2}{|c|}{$-98.80(-94.26 ; 291.86) 0.00$} \\
\hline MENAN_DHAPQ_AL_2011 & - & $1.00(-0.96 ; 2.96)$ & 13.91 \\
\hline YAVO_DHAPQ_AL_2011 & - & $1.00(-0.96 ; 2.96)$ & 13.91 \\
\hline FAYE_ASMQ_AL_2010 & - & $1.00(-0.90 ; 2.90)$ & 14.84 \\
\hline NDIAYE_ASAQ_AL_2011 & • & $0.99(-0.91 ; 2.88)$ & 14.87 \\
\hline SYUA_ASAQ_AL_DHAPQ_2013 & - & $0.99(-0.97 ; 2.95)$ & 13.91 \\
\hline TINE_ASMQ_AL_2012 & • & $0.99(-0.95 ; 2.94)$ & 14.10 \\
\hline Overall (l-squared $=0.0 \% ; \mathrm{p}=0.995)$ & & $1.00(0.27 ; 1.73)$ & 100.00 \\
\hline-292 & 0 & 292 & \\
\hline
\end{tabular}

Figure 1: In the intent-to-treat analysis, APCR was higher for DHPQ, ASMQ for adults, ASAQ for children and ASAQ for adults than for AL; but the differences were not statistically significant.

\begin{tabular}{|c|c|c|c|}
\hline Study ID & & ES $(95 \% \mathrm{Cl})$ & $\begin{array}{c}\% \\
\text { Weight }\end{array}$ \\
\hline FAYE_ASAQ_AL_2010 & - & $0.97(-0.95 ; 2.90)$ & 10.77 \\
\hline FAYE_ASAQ_AL_2012 & t & $96.60(-85.87 ; 279.07$ & 070.00 \\
\hline MENAN_DHAPQ_AL_2011 & - & $0.98(-0.88 ; 2.83)$ & 11.58 \\
\hline YAVO_DHAPQ_AL_2011 & . & $0.95(-0.96 ; 2.86)$ & 10.93 \\
\hline FAYE_ASMQ_AL_2010 & - & $0.96(-0.87 ; 2.80)$ & 11.83 \\
\hline NDIAYE_ASAQ_AL_2011 & . & $0.98(-0.90 ; 2.87)$ & 11.23 \\
\hline SYLLA_ASAQ_AL_DHAPQ_2013 & . & $0.97(-0.97 ; 2.91)$ & 10.63 \\
\hline FAYE_ASAQ_AL_2007 & . & $1.00(-0.96 ; 2.96)$ & 10.39 \\
\hline NDIAYE_2009 & - & $0.95(-0.91 ; 2.81)$ & 11.54 \\
\hline TINE_ASMQ_AL_2012 & • & $0.96(-0.94 ; 2.85)$ & 11.10 \\
\hline Overall (I-squared $=0.0 \%, p=0.9995$ ) & I & $0.97(0.34 ; 1.60)$ & 100.00 \\
\hline-279 & 0 & 279 & \\
\hline
\end{tabular}

Figure 2: On a per protocole basis (PP), the ACPR was higher with ASAQ for children and ASAQ for adults than with routine ASAQ and it was identical between AL and DHAPQ and ASMQ; the difference was not significant.

\begin{tabular}{|c|c|c|}
\hline Study ID & ES $(95 \% \mathrm{Cl})$ & $\begin{array}{c}\% \\
\text { Weight }\end{array}$ \\
\hline FAYE_ASAQ_AL_2012 & $\rightarrow 0.35(-0.42 ; 1.11)$ & 1.01 \\
\hline MENAN_DHAPQ_AL_2011 & $0.20(-0.21 ; 0.61)$ & 3.48 \\
\hline YAVO_DHAPQ_AL_2011 & $0.06(-0.14 ; 0.26)$ & 15.34 \\
\hline FAYE_ASMQ_AL_2010 & $0.21(-0.18 ; 0.60)$ & 3.83 \\
\hline SYLLA_ASAQ_AL_DHAPQ_2013 & $0.04(-0.13 ; 0.20)$ & 21.74 \\
\hline TINE_ASMQ_AL_2012 & $0.03(-0.08 ; 0.13)$ & 54.60 \\
\hline Overall (I-squared $=0.0 \%, p=0.854$ ) & $0.05(-0.03 ; 0.13)$ & 100.00 \\
\hline-1.11 & 1.11 & \\
\hline
\end{tabular}

Figure 3: At D1, persistent fever was higher in patients treated with another ACT.
Fever clearance: At D1, persistent fever was higher in patients treated with another ACT (Figure 3). At D2, thermic clearance was better with DHAPQ and DHAPQTMP; persistent fever was more found in ASAQ and ASMQ treated children than in children treated with AL. At D3, all patients were without fever regardless of the combination used.

Parasite clearance: On D1 into treatment, the proportion of patients with persisting parasites was larger among the patients treated with ASAQ DHAPQ and ASMQ (Figure 4). On D2, parasite clearance was better for all ACTs as compared to AL, except for patients treated with the combination DHA-PQ-TMP (Figure 5).

\begin{tabular}{|c|c|c|c|}
\hline Study ID & & ES $(95 \% \mathrm{Cl})$ & $\begin{array}{c}\% \\
\text { Weight }\end{array}$ \\
\hline MENAN_DHAPQ_AL_2011 & $\rightarrow-$ & $0.19(-0.25 ; 0.63)$ & 20.94 \\
\hline FAYE_ASMQ_AL_2010 & $\rightarrow$ & $0.18(-0.09 ; 0.45)$ & 52.66 \\
\hline SYLLA_ASAQ_AL_DHAPQ_2013 & & $0.75(-0.56 ; 2.06)$ & 2.31 \\
\hline FAYE_ASAQ_AL_2007 & $\longrightarrow$ & $0.74(0.34 ; 1.15)$ & 24.09 \\
\hline YAVO_DHAPQ_AL_2011 & & Excluded & 0.00 \\
\hline Overall (I-squared $=49.5 \%, p=0.115$ ) & & $0.33(0.13 ; 0.53)$ & 100.00 \\
\hline-2.06 & $\begin{array}{l}1 \\
0\end{array}$ & 2.06 & \\
\hline
\end{tabular}

Figure 4: On D1 into treatment, the proportion of patients with persisting parasites was larger among the patients treated with ASAQ, DHAPQ and ASMQ.

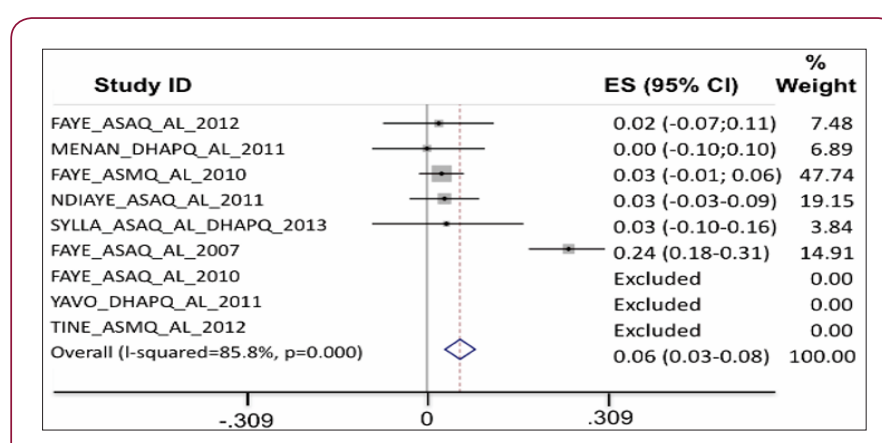

Figure 5: On D2, parasite clearance was better for all ACTs as compared to AL, except for patients treated with the combination DHA-PQ-TMP.

Gametocyte carriage: On D1, the gametocyte carriage was lower for AL treated patients than for those treated with ASAQ and ASMQ (Figure 6). On D2, the carriage was similar for all treatment groups analyzed.

\begin{tabular}{|c|c|c|}
\hline Study ID & ES $(95 \% \mathrm{Cl})$ & $\begin{array}{c}\% \\
\text { Weight }\end{array}$ \\
\hline FAYE_ASAQ_AL_2010 & $0.03(-0.03-0.09)$ & 12.63 \\
\hline FAYE_ASAQ_AL_2012 & $0.00(-0.03-0.03)$ & 67.26 \\
\hline FAYE_ASMQ_AL_2010 & $0.06(-0.02-0.13)$ & 7.47 \\
\hline FAYE_ASAQ_AL_2007 & $0.00(-0.06-0.06)$ & 12.63 \\
\hline MENAN_DHAPQ_AL_2011 & Excluded & 0.00 \\
\hline YAVO_DHAPQ_AL_2011 & Excluded & 0.00 \\
\hline Overall (1-squared $=0.0 \%, \mathrm{p}=0.44$ ) & $0.01(-0.01-0.03)$ & 100.00 \\
\hline-134 & 134 & \\
\hline
\end{tabular}

Figure 6: On D1, the gametocyte carriage was lower for AL treated patients than for those treated with ASAQ and ASMQ. 
Clinical tolerance: On D1 of the treatment, all patients treated with ACTs developed more significant side effects than those treated with AL. On D2 and D3, only the group treated with ASMQ developed clinical signs more significant than in the AL group.

\section{Discussion}

In this study, we have analyzed and compared 11 clinical trials carried out in various sites in Senegal. All of them were published. This allowed us to assess the effectiveness and tolerance of artemisin-based therapeutic combinations which, for some of them, have been compared with arthemether-lumefantrine (AL). As the only combination qualified certified by the WHO at the time of the study, AL was the reference combination for it. All these evaluations were based on the WHO 2001 protocol updated in 2003 and 2006. This protocol requires monitoring over 28 successive days to measure clinical and parasitological effectiveness after correction by PCR. At inception, all treatment groups were equal in respect of clinical, biological and sociodemographic data.

\section{Efficacy}

The combinations that are in use today are very effective against uncomplicated Plasmodium Falciparum malaria. Indeed, the study carried out in Keur Soce in 2013 revealed rates of adequate clinical and parasitological response (ACPR) before correction by PCR of $99.67 \%$ for l'ASAQ and $96.02 \%$ for DHAPQ against $98,31 \%$ for AL in intent-to-treat (ITT). In per protocole (PP) mode, the rates reached $99.41 \%$ for ASAQ, 99.38\% for DHAPQ against $99.42 \%$. After correction by PCR, the effectiveness rates were established at 97.22\%, 96.59\% and 98.88\% respectively for ASAQ, DHAPQ and AL in ITT. In PP, they reached $99.41 \%$ for l'ASAQ as against $100 \%$ for DHAPQ and AL. Another study carried out in Guédiawaye, Kaolack and Richard Toll in 2007 revealed effectiveness rates of $97.50 \%$ for ASAQ $97.90 \%$ for ASMQ and $100 \%$ for AL in ITT. The same rates reached $100 \%$ for the same three combinations after correction with PCR. These results confirm data previously published after similar clinical trials. In Asia, well before the studies performed in Senegal, Denis and al found in 2002 in Cambodia an ACPR of $96.9 \%$ for Dihydroartemisinin/Piperaquine on D28 [10].

A randomized comparative study carried out in Thailand on the same year found an APCR as high as $100 \%$ in the ASMQ group and 99\% in the DAHPQ group on D28 [11]. In Senegal, Brasseur et coll studied the efficacy and tolerance of the Artesunate-Amodiaquine combination within the framework of a clinical trial carried out in Casamance (Southern region of Senegal) between 2000 and 2005. 966 patients were involved in this study and they were monitored until D28. The average ACPR on D28 over the six years was established at $94.6 \%$, with variations between $88.5 \%$ and $96.7 \%$ [12]. As for ASAQ, it has been shown to be equal to AL both in adults and in children, whether in a single dose or two intakes a day. Other studies performed in Africa, for example Falade Co et al in 2008 in Nigeria [13] confirm those findings. In a randomized comparative study carried out in Uganda in 2006 to measure the effectiveness and tolerance of the Dihydroartmisin/Piperaquine combination versus Artéméther/Luméfantrine for the treatment of uncomplicated P. falciparum malaria, Adoke Y et al found lower
ACPR rates on D28: 86.5\% for DHAPQ against 65.5\% for AL. The rate of therapeutic failure was established at 3.9\% for DHAPQ versus $17.3 \%$ for AL [14].

In Asia, Ratcliff $\mathrm{A}$ et al carried, in an extensive clinical studies in Thailand, Cambodia, Vietnam and China, found the Dihydroartemisinine/piperaquine combination to be slightly more effective than l'Arteméther/Lumefantrine in multi-resistance areas [15]. DHAPQ TMP is a triple combination (the first ever used to treat malaria) which has been tested and compared to AL. Its efficacy and non-inferiority to AL were demonstrated by a 95\% ACPR rate in ITT and $98.7 \%$ in PP as against $95 \%$ in ITT and $100 \%$ in PP for AL before correction by PCR. After correction, the ACPR rate was $98 \%$ for DHAPQ TMP and $95 \%$ for AL in ITT and 100\% for both in PP. Using DHPQ as a basis combination and adding trimethoprime to it was decided on the basis of pre-clinical studies which demonstrated the effectiveness of that association. Only a few human trials of the Dihydroartemisinine-Piperaquine phosphate-Trimethoprime combination have been carried out: in 2002, the effectiveness and tolerance of DHAP-T vs Artesunate-Mefloquine were assessed in Thailand, in an open-label randomized trial on 352 adults with acute uncomplicated Plasmodium falciparum malaria.

The cure rate on Day 28 was found to be $97 \%$ in both groups [16]. Still in Asia, in 2004, Tran TH et al assessed the effectiveness and safety of the combinations Dihydroartemisinin - PiperaquineTrimethoprime, Dihydroartemisinin-Piperaquine and ArtesunateMefloquine as treatment for uncomplicated Plasmodium falciparum malaria in a study carried out in Vietnam on 400 patients. They found ACPR rates in ITT at D56 as high as $97.4 \%$ in the DHA-P-T group, $98.6 \%$ in the DHA-P and the ASMQ groups [17]. Other studies relating to the effectiveness and safety of the Artesunate/Mefloquine, Artesunate/Amodiaquine and Artesunate/ Sulfadoxine-pyrimethamine combinations in the treatment of uncomplicated P. falciparum malaria in Africa, Asia and South America showed ACPR rates on D28 higher than 95\% [18-22].

\section{Fever Clearance}

Fever disappeared gradually in the groups treated with the various combinations tested, with a total clearance observed on D1 or D2 into the treatment. On D3, fever had disappeared from all patients. Adjei G O et Alen 2008 achieved a rapid elimination of fever 24 hours into treatment with AS-AQ as against 48 hours intro treatment with AL [23]. As to other ACT combinations, the surveys carried out in other countries have shown their effectiveness in eliminating fever $[24,25]$.

\section{Parasite Clearance}

Within the context of growing resistance to artemisinin derivatives, especially in Asia, this criterion is important to show a lower sensitivity of the parasite to ACTs. In fact, a longer parasite clearance time is one of the first steps towards resistance. Parasite clearance was achieved on D1 or D2 with AL and on D2 with ASAQ in most surveys. Other authors who compared ASAQ and AL indicated similar results [13,26,27]. With DHAPQ TMP, clearance was observed on D2 whereas for ASMQ and DHAPQ persistent parasitemia was observed in some patients until D7. 


\section{Clinical Safety}

From a clinical point of view, the treatments were well tolerated by patients with similar profiles. The number of adverse events recorded in each group was minimal and they did not require a interruption of treatment. However, the side-effects noted were more intense in the study which compared ASMQ and AL in adults.

\section{Conclusion}

ACTs are very effective, and they can be significant contributor to the management of uncomplicated P. falciparum malaria. The ASAQ ASMQ, DHAPQ and DHPQTMP combinations are as effective and safe as the Arthemeter/Lumefantrin combination. However, the following recommendations need to be made towards a greater effectiveness of the current malaria control strategies:

a) These new molecules are effective against resistant strands and they must be made more financially accessible.

b) Every malaria case must be biologically confirmed by thick smear or rapid diagnostic test before treatment with ACT.

c) The chemosensitivity of $P$ falciparum strands to the various ACTs must be regularly monitore.

d) Pharmacovigilance must be maintained to allow for the detection of unexpected adverse events.

\section{References}

1. OMS (2004) Evaluation et surveillance de 1 for the detection of unexpected adverse events. re treatment with ACT $r$ the following recommendation WHO.

2. (2015) The millennium development goals report. United Nations New York, USA.

3. Programme des Nations Unies pour le Développement (PNUD). Objectifs de development durables (ODD).

4. (2016) WHO. Global Malaria Programme. World malaria report p. 22-23.

5. OMS (2001) Utilisation des antipaludiques : Rapport d'une consultation informelle de l'OMS.

6. OMS (2001) Les Combinaisons thérapeutiques antipaludiques : Rapport d'une consultationtechnique de l'OMS, 4-5 avril 2001.

7. (2011) OMS / Roll Back Malaria. Plan mondial de maitrise de la résistance à l'Artémisinine / Global Plan for Artemisinin Resistance (GPARC) Résumé d'orientation.

8. (2006) Ministère de la Santé et de la Prévention Médicale. Directives pour la prise en charge du paludisme au Sénégal. Programme National de Lutte contre le Paludisme, janvier.

9. (2013) PNLP Plan stratégique. Plan stratégique de lutte contre le paludisme au Senegal 2011-2015. Doc ministre de la sante et de l'action sociale aout 2013.

10. Denis MB, Davis TM, Hewitt S, Incardona S, Nimol K, et al. (2002) Efficacy and safety of dihydroartemisinin-piperaquine (Artekin) in Cambodian children and adults with uncomplicated falciparum malaria. Clin Infect Dis 35(12): 1469-1476.

11. Tangpukdee N, Krudsood S, Thanachartwet W, Chalermrut K, Pengruksa C, et al. (2005) An open randomized clinical trial of Artekinvsartesunatemefloquine in the treatment of acute uncomplicated falciparum malaria. Southeast Asian J Trop Med Public Health 36(5): 1085-1091.

12. Brasseur P, Agnamey P, Gaye O, Vaillant M, Walter RJ, et al. (2007) Efficacy and safety of artesunate plus amodiaquine in routine use for the treatment of uncomplicated malaria in Casamance, southern Senegal. Malar J 6: 150.

13. Falade CO, Ogundele AO, Yusuf BO, Ademowo OG, Ladipo SM (2008) High efficacy of two artemisinin-based combinations (artemetherlumefantrine and artesunate plus amodiaquine) for acute uncomplicated malaria in Ibadan, Nigeria. Trop Med Int Health 13(5): 635-643.

14. Adoke Y, Grant D, Morses RK, Ambrose T, Myers L, John BR, et al. (2008) Artéméther/Luméfantrineversusdihdroartemisinin/piperaquine for treating uncomplicated malaria. PLoS ONE (6): e2339.

15. Ratcliff A, Siswantoro H, Kenangalem E, Maristela R, Wuwung RM et al. (2007) Two fixed-dose artemisinin combinations for drug-resistant falciparum and vivax malaria in Papua, Indonesia: an open-label randomised comparison. Lancet 369(9563): 757-765.

16. Wilairatana P, Krudsoods S, Chalermrut K, Pengruksa C, Srivilairit S, et al. (2002) An open randomized clinical trial of Artecomvs ArtesunateMefloquine in the treatment of acute uncomplicated falciparum malaria in Thailande. Southeast Asian J Trop Med Public Health 33(3): 519-524.

17. Tran TH, Dolecek C, Pham PM, Nguyen TD, Nguyen TT et al. (2004) Dihydroartemisinin-piperaquine against multidrug-resistant Plasmodium falciparum Malaria in Vietnam: randomized clinical. Lancet 363(9402): 18-22

18. Bergal S, Nores JM, Rosenheim M, Peignot JF, Paraf F, et al. (1986) Paludisme: Préparation aux certificats et au concours de l'internat. Editions SPECIA.

19. Djimde AA, Fofana B, Sagara I, Sidibe B, Toure S, et al. (2008) Efficacy, safety and selection of molecular markers of drug resistance by two ACTs in Mali. Am J Trop Med Hyg 78(3): 445-461

20. Martin M, Alaribe A, Ejemot R, Oyo-Ita A, Ekenjoku J, et al. (2006) Artemether-Lumantrine versus Artesunate plus Amodiaquine for treating uncomplicated childhood malaria in Nigeria: randomized controlled trial. Malar J 5: 43.

21. Osorio L, Gonzalez L, Olliaro, Walter R (2007) Artemisinin-based combination therapy of uncomplicated Plasmodium falciparum malaria in Colombia. Malar J 6: 25.

22. Vugt MV, Wilairatana P, Gemperli B, Gathmann I, Phaipun L, et al. (1999) Efficacy of six doses of artemether-lumefantrine(benflumetol) in multidrug-resistant Plasmodium falciparum malaria. Am J Trop Med Hyg Jun 60(6): 936-942.

23. Adjei GO, Kurtzhals JA, Rodrigues OP, Alifrangis M, Hoegberg LC, et al. (2008) Jun 11 Amodiaquine-artesunate contre artemether-luméfantrine pour le paludisme peu compliqué chez les enfants ghanéens: une éfficacité randomisée et un procés de sécurité avec une suite d'année. Malar J 7: 127

24. Diatta M (2008) Etude randomisée comparative pour mesurer l'efficacité et la tolérance de l'association Dihydroartémisinine/Pipéraquine (DuoCotecxin $\AA$ ) versus Artéméther/Luméfantrine (Coartem $®$ ) dans le traitement du paludisme non compliqué à Plasmodium falciparum au Sénégal (Touba). Thèse Pharm. $N^{\circ} 111$.

25. Price RN, Nosten F, Luxemburger C, Van Vugt M, Phaipun L, et al. (1998) Artesunate/mefloquine treatment of multi-drug resistant falciparum malaria. Trans R Soc Trop Med Hyg 92(1): 122

26. Meremikwum M, Alaribe A, Ejemot R, Oyp Ita A, Ekenjoku J, et al. (2006) Artemether-Lumefantrine versus Artesunate plus Amodiaquine for treating uncomplicated childhood malaria in Nigeria: randomized controlled trial. Malar J 5: 43.

27. Ndayiragije A, Niyungeko D, Karenzo J, Niyungeko E, Barutwanayo M, et al. (2004) Efficacité de combinaisons thérapeutique avec des dérivés de l'artémisinine dans le traitement de l'accès palustre non compliqué au Burundi. Trop Med in Health 9(6): 673-679. 


\section{ISSN: 2574-1241}

DOI: 10.26717/BJSTR.2018.08.001674

Papa Ndiaye. Biomed J Sci \& Tech Res

(c) 9 This work is licensed under Creative

Submission Link: https://biomedres.us/submit-manuscript.php

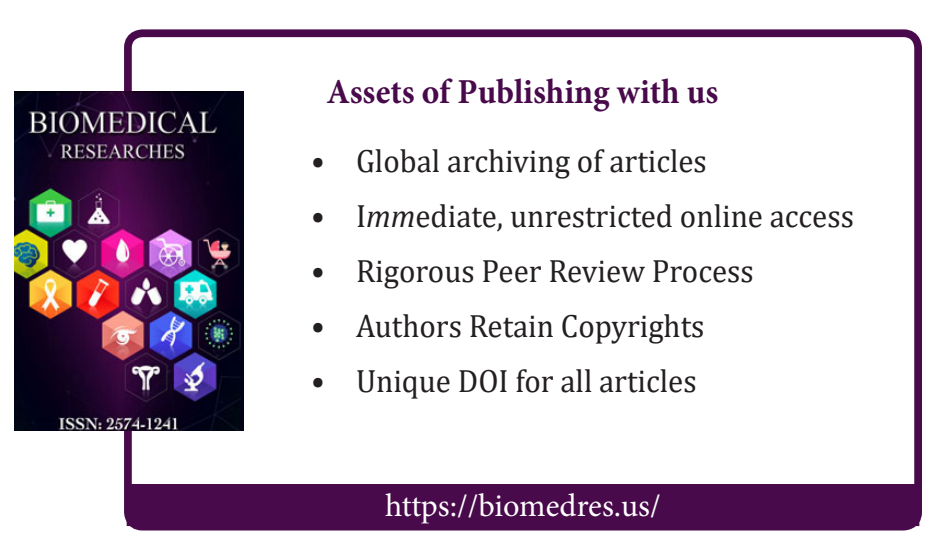

